honoraria from Pfizer outside this work, Giorgio Carlino Consultant for: $G$ Carlino had has received consultancy fees from Pfizer, Janssen, AbbVie, MSD, BMS., Romano Bucci Consultant for: $\mathrm{R}$ Bucci has received consultancy fees and/or speaker honoraria from Pfizer, Sanofi, MSD, BMS, Speakers bureau: $\mathrm{R}$ Bucci has received consultancy fees and/or speaker honoraria from Pfizer, Sanofi, MSD, BMS, Angelo Semeraro Speakers bureau: A Semeraro has received speaker honoraria from Sanofi, Roche, AbbVie, BMS, MSD, Novartis, Antonio Marsico: None declared, Daniela Mazzotta: None declared, Paola Chiara Francesca Falappone Consultant for: PC Falappone had received consultancy fees and/or speaker honoraria from Amgen, Abbott, MSD, BMS, outside this work, Speakers bureau: PC Falappone had received consultancy fees and/or speaker honoraria from Amgen, Abbott, MSD, BMS, outside this work, Florenzo lannone Consultant for: $F$ lannone has received consultancy fees and/or speaker honoraria from Pfizer, AbbVie, MSD, BMS, Novartis, Lilly, UCB outside this work, Speakers bureau: $F$ lannone has received consultancy fees and/or speaker honoraria from Pfizer, AbbVie, MSD, BMS, Novartis, Lilly, UCB outside this work

DOI: 10.1136/annrheumdis-2019-eular.5817

\section{AB0291B A COMPARISON OF THE JOINTS ULTRASONOGRAPHY IN THE PATIENTS WITH RHEUMATOID ARTHRITIS TREATED BY BIOLOGICAL AGENTS AND THE CORRESPONDING SYNOVIAL HISTOLOGICAL FINDINGS}

Asami Abe ${ }^{1}$, Hajime Ishikawa ${ }^{1}$, Kunihiko Wakaki ${ }^{2}{ }^{1}$ Niigata Rheumatic Center, Rheumatology, Shibata, Japan; ${ }^{2}$ Niigata Prefectural Shibata Hospital, Pathology, Shibata, Japan

Background: In the treatment of Rheumatoid arthritis (RA), early diagnosis and early treatment with tight control have become increasingly important with the advent of biological therapy. Ultrasonography (US) of the various joints enables the real-time evaluation of synovial hypertrophy, effusion and bone erosion, Power Doppler (PD) ultrasound is able to identify both subclinical synovitis and early erosive disease.

Objectives: The objectives of this study were to investigate whether the image of US at the operated joint reflect synovium histological findings or clinical indicators, and to compare the results in the patient treated by non-biological agent (NonBio) and biological agent (Bio)

Methods: RA related orthopaedic surgery was performed at 1287 joints including 17 shoulders, 160 knees, 96 elbows, 378 wrists, 364 fingers, 38 ankles and 234 toes during the period between January 2011 and December 2018 at our rheumatic center. Preoperatively, ultrasound evaluations were performed and grade of PD signal was determined at the part with the highest signal. PD signal consists 4 grades from grade 0 to 3. The operations were performed within one week after the ultrasound evaluations. Rooney score of the synovium histology, DAS28-ESR (4), MMP-3, CRP were investigated. Rooney score represents the histologic features in the synovium of RA. It includes 6 features i.e. synoviocytes hyperplasia, fibrosis, proliferating blood vessels, perivascular infiltrates of lymphocytes, focal aggregates of lymphocytes, diffuse infiltrates of lymphocytes. They were scored separately on a scale of 1-10. The treatments that the patients received included: biological agents (Bio) $(n=38930 \%)$, etanercept (ETN) $(n=102)$, tocilizumab (TCZ) $(n=105)$, infliximab (IFX) ( $n=29)$, adalimumab (ADA) $(n=28)$, certlizumab pegol (CZP) ( $n=12)$, golimumab (GLM) ( $n=56)$, and abatacept (ABT) $(n=55)$, tofacitinib (TOF) $(n=2)$.

Results: PD signal $(0.97 \pm 0.88)$, DAS28 $(3.23 \pm 1.13), \operatorname{CRP}(0.5 \pm 1.25 \mathrm{mg} / \mathrm{dL})$, MMP-3 $(119.0 \pm 119.0 \mathrm{ng} / \mathrm{mL})$ and Rooney score $(23.5 \pm 7.7)$ in the patients treated with Bio were significantly lower than those $(1.39 \pm 0.95,3.66 \pm 1.07$, $0.78 \pm 1.37 \mathrm{mg} / \mathrm{dL}, \quad 146.3 \pm 150.0 \mathrm{ng} / \mathrm{dL}, 28.5 \pm 9.26)$ in the patients treated with NonBio. Rooney score fibrosis $(9.57 \pm 1.44)$ in patients treated with Bio was significantly higher than those $(8.64 \pm 2.48)$ in patients treated by NonBio. Rooney score, synoviocyte hyperplasia (1.16 \pm 1.26$)$ and three items of lymphocyte $(1.59 \pm 2.91,1.68 \pm 2.54,1.11 \pm 2.20)$ in patients treated with Bio were lower than those $(1.72 \pm 1.30,3.79 \pm 3.56,3.73 \pm 3.53,2.40$ \pm 2.98 ) in patients treated with NonBio. TCZ, ADA, ABT and IFX had some significant differences for Rooney score and Rooney item score between the patients treated with NonBio.

Conclusion: The activity of RA synovitis at operated site was suppressed in patients treated with Bio. There were some differences in clinical data, histological score, PD signal and DAS28 among Bio.

Disclosure of Interests: None declared

DOI: 10.1136/annrheumdis-2019-eular.1159

\section{AB0291C REMISSION IN RHEUMATOID ARTHRITIS: CONSIDERING NEW CUT-POINTS OF COMPOSITE DISEASE ACTIVITY INDICES ACCORDING TO THE ABSENCE OF SYNOVITIS BY ULTRASONOGRAPHY}

Ana Bertoli ${ }^{1}$, Marcelo Audisio ${ }^{2}$, Mariana Benegas ${ }^{3}$, Maximiliano Bravo ${ }^{4}$, Erika Catay ${ }^{4}$, Tomás Cazenave ${ }^{5}$, Claudia Hartvig ${ }^{6}$, Paula Kohan ${ }^{7}$, Josefina Marin ${ }^{8}$, Victoria Martire ${ }^{9}$, David Navarta ${ }^{10}$, Javier Rosa ${ }^{8}$, Marcos Rosemffet ${ }^{5}$, Santiago Ruta ${ }^{11}$, Lida Santiago ${ }^{12}$, Ethel Saturansky ${ }^{3}$, Juan Soldano ${ }^{6}$, Walter Spindler ${ }^{13}$, Johana Zacariaz ${ }^{8}$, ECOSAR. Sociedad Argentina de Reumatología.. I'Instituto Reumatologico Strusberg, Rheumatology, Córdoba, Argentina; ${ }^{2}$ Humana Instituto Médico Privado, Córdoba, Argentina; ${ }^{3}$ Psoriahue, Lomas de Zamora, Argentina; ${ }^{4}$ Consultorios Moreno, Formosa, Argentina; ${ }^{5}$ Instituto de Rehabilitación Psicofísica, Buenos Aires, Argentina; ${ }^{6}$ Hospital Provincial de Rosario, Rosario, Argentina; ' Dr. Enrique Tornú Hospital, Buenos Aires, Argentina; ${ }^{8}$ Hospital Italiano de Buenos Aires, Buenos Aires, Argentina; ${ }^{9}$ Instituto Medico Platense, La Plata, Argentina; ${ }^{10}$ Hospital Dr. Marcial Quiroga, San Juan, Argentina; ${ }^{11}$ Hospital Italiano La Plata, La Plata, Argentina; ${ }^{12}$ Organización Médica de Investigación, City of Buenos Aires, Argentina; ${ }^{13}$ Centro Medico Privado de Reumatologia, San Miguel de Tucumán, Argentina

Background: Achieving the state of remission in RA according to currently available criteria does not entirely prevent patient from radiological progression. On the contrary, the presence of subclinical synovitis by means of ultrasonography (US) correlates with radiological progression. Objectives: To stablish cut-off values of composite disease activity indices (DAS28-CRP, DAS28-ESR, CDAI and SDAI) that adjust better to remission according to the absence of synovitis by US.

Methods: Observational, cross-sectional study that included 126 patients with diagnosis of RA who were in remission or low disease activity (DAS-ESR 53.2). For each patient we calculated the DAS28-ESR, DAS28-CRP, CDAI, SDAI. For US assessment we used the 12 joint simplified score. We considered the state of remission by US if synovia hypertrophy $(\mathrm{SH}) \leq 1$ and power Doppler $(\mathrm{PD})$ signal= 0 in all joints. The cut-off values of the disease activity indices to define remission according the proposed US definition of remission were determined by ROC curves. Results: Patients had a median (ICR) DAS28-ESR of $2.32(0.60)$ $\mathrm{DAS28}-\mathrm{CRP}=1.89$ (0.55), $\mathrm{CDAl}=3.56$ (3.0) and $\mathrm{SDAl}=3.0$ (4.7). The sensitivity, specificity, positive and negative predictive value of each of the selected cut-off points for the DAS28-CRP, DAS28-ESR, CDAI and SDAl for the definition of remission are shown in the Table.

Conclusion: In this study we describe new cut points of the different composite disease activity indices for the definition of remission. These cut points improve the accuracy of the definition of remission. However they differ scarcely from the reference values making its impact uncertain in the long term management of patients with RA. Longitudinal studies are, therefore, warranted in order to elucidate the performance of these new cut points.

\section{REFERENCE}

[1] Aletaha D, Smolen JS. Clin Exp Rheumatol 2006; 24(6 Suppl 43): S-4551.

\begin{tabular}{|c|c|c|c|c|}
\hline $\begin{array}{l}\text { Cut-off } \\
\text { values }\end{array}$ & $\begin{array}{c}\text { Sensitivity\% } \\
\text { (IC95\%) }\end{array}$ & $\begin{array}{c}\text { Specificity\% } \\
\text { (IC95\%) }\end{array}$ & $\begin{array}{l}\text { PPV\% } \\
\text { (IC95\%) }\end{array}$ & $\begin{array}{l}\text { NPV\% } \\
\text { (IC95\%) }\end{array}$ \\
\hline \multicolumn{5}{|c|}{ Taking as reference SH definition of remission. } \\
\hline DAS28-ESR & $81(70-88)$ & $50(37-63)$ & $68(58-77)$ & $66(51-78)$ \\
\hline$\leq 2.6$ & $65(54-75)$ & $63(56-73)$ & $70(58-80)$ & $58(45-69)$ \\
\hline \multicolumn{5}{|l|}{$\leq 2.5$} \\
\hline DAS28-CRP & $86(76-92)$ & $26(16-39)$ & $61(51-70)$ & $58(39-76)$ \\
\hline$\leq 2.6$ & $57(45-68)$ & $55(41-68)$ & $63(51-74)$ & $48(36-61)$ \\
\hline \multicolumn{5}{|l|}{$\leq 2.0$} \\
\hline CDAI & $61(50-72)$ & $74(61-84)$ & $76(64-85)$ & $59(47-70)$ \\
\hline$\leq 2.8$ & $60(48-70)$ & $74(61-84)$ & $75(63-85)$ & $58(46-69)$ \\
\hline \multicolumn{5}{|l|}{$\leq 2.1$} \\
\hline SDAI & $52(40-63)$ & $72(59-82)$ & $71(58-82)$ & $53(42-64)$ \\
\hline$\leq 3.3$ & $52(41-64)$ & $80(68-89)$ & $78(64-88)$ & $55(44-66)$ \\
\hline \multicolumn{5}{|l|}{$\leq 3.0$} \\
\hline \multicolumn{5}{|c|}{ Taking as reference the PD definition of remission. } \\
\hline DAS28-ESR & $71(61-80)$ & $43(28-59)$ & $76(66-84)$ & $37(25-52)$ \\
\hline$\leq 2.6$ & $58(48-68)$ & $60(44-74)$ & $79(68-87)$ & $36(25-48)$ \\
\hline \multicolumn{5}{|l|}{$\leq 2.5$} \\
\hline DAS28-CRP & $80(71-87)$ & $17(8-33)$ & 72 (62-79) & $25(18-45)$ \\
\hline$\leq 2.2$ & $64(53-73)$ & $56(40-71)$ & $79(68-87)$ & $38(26-52)$ \\
\hline \multicolumn{5}{|l|}{$\leq 1.96$} \\
\hline CDAI & $55(44-64)$ & $47(58-86)$ & 85 (73-92) & $38(28-50)$ \\
\hline$\leq 2.8$ & $53(43-63)$ & $74(58-86)$ & 84 (73-92) & $38(27-50)$ \\
\hline \multicolumn{5}{|l|}{$\leq 2.15$} \\
\hline SDAI & $46(36-56)$ & $71(55-84)$ & 81 (68-89) & $34(24-489)$ \\
\hline$\leq 3.3$ & $46(36-57)$ & $82(67-92)$ & $87(74-94)$ & $33(28-49)$ \\
\hline$\leq 3.0$ & & & & \\
\hline
\end{tabular}


Disclosure of Interests: None declared

DOI: 10.1136/annrheumdis-2019-eular.4411

\section{AB0291D $\quad$ PREDICTORS OF NEW BONE EROSION IN RHEUMATOID ARTHRITIS PATIENTS RECEIVING CSDMARDS: ANALYSIS OF DATA FROM THE DRIVE AND DESIRABLE STUDIES}

Tsutomu Takeuchi ${ }^{1}$, Satoshi Soen ${ }^{2}$, Naoki Ishiguro ${ }^{3}$, Hisashi Yamanaka ${ }^{4}$, Sakae Tanaka ${ }^{5}$, Makiko Kobayashi ${ }^{6}$, Naoki Okubo ${ }^{6}$, Takaya Nitta ${ }^{6}$, Yoshiya Tanaka ${ }^{7}{ }^{1}$ Keio University, Tokyo, Japan; ${ }^{2}$ Nara Hospital, Kinki University School of Medicine, Ikoma, Japan; ${ }^{3}$ Nagoya University, Nagoya, Japan; ${ }^{4}$ Tokyo Women's Medical University, Tokyo, Japan; ${ }^{5}$ The University of Tokyo, Tokyo, Japan; ${ }^{6}$ Daiichi Sankyo Co., Ltd., Tokyo, Japan; ${ }^{7}$ University of Occupational and Environmental Health, Kitakyusyu, Japan

Background: Suppression of joint destruction is an important target for the treatment of rheumatoid arthritis (RA). Most previous studies have proposed prediction models for joint destruction to detect the risk of rapid radiographic progression (change in $\mathrm{mTSS} \geq 5$ ). As joint destruction progresses irreversibly, even slight progression of joint destruction might impact the prognosis. Therefore, our study focused on the onset of new bone erosion in RA patients.

Objectives: To clarify predictors for new bone erosion in RA patients treated with csDMARDs.

Methods: Predictive factors were analyzed using data from the placebo groups of the DRIVE [1] and DESIRABLE [2] studies, which were 12month, randomized, double-blind, phase 2 and 3 trials for evaluating the efficacy of denosumab in RA patients. New bone erosion was defined as change from baseline in erosion score (ES) $\geq 1.0$ at 12 months, which was assessed as "progressed" by two readers. In addition to newly emerging erosion, new bone erosion also included enlargement of erosion size which is the result of new erosion at a site adjacent to an existing bone erosion. To evaluate predictors for new bone erosion, a logistic regression model was applied. Significant predictors ( $p$ value of $<0.1$ ) were selected from the univariate analysis and one variable from each correlated pair that showed significance was removed. Multivariate analyses were performed using the selected predictors.

Results: In a total of 306 patients, baseline DAS28-CRP (mean \pm SD) was $3.58 \pm 1.03$. New bone erosion was observed in 90 patients $(29.4 \%)$

In the univariate analysis, female sex, anti-CCP antibody positivity, rheumatoid factor (RF) positivity, tender joint count $(T J C) \geq 6, C R P \geq 0.3 \mathrm{mg} / \mathrm{dL}$, erythrocyte sedimentation rate $(E S R) \geq 28 \mathrm{~mm} / \mathrm{h}$, and baseline $E S \geq 3$ were identified as significant predictors for new bone erosion. RF and ESR were not included in the multivariate analysis because they were strongly correlated with anti-CCP antibody and $\mathrm{CRP}$, respectively. In the multivariate analyses, female sex, anti-CCP antibody positivity, TJC $\geq 6$, $\mathrm{CRP} \geq 0.3 \mathrm{mg} / \mathrm{dL}$, and baseline $\mathrm{ES} \geq 3$ were identified as predictors for the development of new bone erosion.

Abstract AB0291D Table 1. Univariate and multivariate analyses for a change in $E S \geq 1.0$

\begin{tabular}{|c|c|c|c|c|c|c|}
\hline \multirow{2}{*}{ Variables } & & \multirow[b]{2}{*}{ Reference } & \multicolumn{2}{|c|}{ Univariate } & \multicolumn{2}{|c|}{ Multivariate } \\
\hline & & & $\begin{array}{c}\text { Odds } \\
\text { ratio }\end{array}$ & $P$ value & $\begin{array}{l}\text { Odds } \\
\text { ratio }\end{array}$ & $P$ value \\
\hline Sex & Female & Male & 1.78 & 0.089 & 1.85 & 0.097 \\
\hline Age & $\geq 65$ years & $<65$ years & 0.79 & 0.423 & & \\
\hline $\begin{array}{l}\text { Duration of } \\
\text { disease }\end{array}$ & $\geq 3$ years & $<3$ years & 1.06 & 0.844 & & \\
\hline Anti-CCP antibody & Positive & Negative & 5.95 & $<0.001$ & 4.01 & $<0.001$ \\
\hline RF & Positive & Negative & 2.67 & $<0.001$ & & \\
\hline Glucocorticoid use & Yes & No & 0.99 & 0.963 & & \\
\hline TJC & $\geq 6$ & $<6$ & 1.66 & 0.047 & 1.70 & 0.060 \\
\hline Swollen joint count & $\geq 10$ & $<10$ & 1.26 & 0.353 & & \\
\hline CRP & $\geq 0.3 \mathrm{mg} / \mathrm{dL}$ & $\begin{array}{c}<0.3 \mathrm{mg} / \\
\mathrm{dL}\end{array}$ & 2.84 & $<0.001$ & 2.02 & 0.013 \\
\hline ESR & $\geq 28 \mathrm{~mm} / \mathrm{h}$ & $<28 \mathrm{~mm} / \mathrm{h}$ & 2.08 & 0.007 & & \\
\hline Baseline ES & $\geq 3$ & $<3$ & 3.32 & $<0.001$ & 2.29 & 0.005 \\
\hline Study & DESIRABLE & DRIVE & 1.16 & 0.602 & & \\
\hline
\end{tabular}

Conclusion: In RA patients whose disease activity was controlled on csDMARDs, positive anti-CCP antibody/RF status, elevated CRP/ESR levels, baseline $E S \geq 3, T J C \geq 6$ and female sex were identified as predictors for new bone erosion.

\section{REFERENCES}

[1] Takeuchi T, et al. Ann Rheum Dis 2016;75:983-90

[2] NCT01973569 (Takeuchi T, et al: in submission)

Disclosure of Interests: Tsutomu Takeuchi Grant/research support from: Astellas Pharma Inc, Chugai Pharmaceutical Co, Ltd., Daiichi Sankyo Co., Ltd., Takeda Pharmaceutical Co., Ltd., AbbVie GK, Asahikase Pharma Corp., Mitsubishi Tanabe Pharma Co., Pfizer Japan Inc., Eisai Co., Ltd., AYUMI Pharmaceutical Corporation, Nipponkayaku Co. Ltd. Novartis Pharma K.K., Grant/research support from: AbbVie, Asahi Kasei, Astellas, AstraZeneca, AYUMI, Bristol-Myers Squibb, Chugai, Daiichi Sankyo, Eisai, Eli Lilly Japan, Janssen, Mitsubishi Tanabe, Nippon Kayaku, Novartis, Pfizer Japan Inc, Taiho, Taisho Toyama, Takeda, Teijin, Grant/research support from: Astellas Pharma Inc., Bristol Myers Squibb, Chugai Pharmaceutical Co., Ltd., Mitsubishi Tanabe Pharma Co., Pfizer Japan Inc., Santen Pharmaceutical Co., Ltd., Takeda Pharmaceutical Co., Ltd., Teijin Pharma Ltd., AbbVie GK, Asahi Kasei Pharma Corp., Taisho Toyama Pharmaceutical Co., Ltd., SymBio Pharmaceuticals Ltd., Janssen Pharmaceutical K.K., Celltrion Inc., Nipponkayaku Co. Ltd., and UCB Japan, Consultant for: Astra Zeneca K.K. Eli Lilly Japan K.K., Novartis Pharma K.K., Mitsubishi Tanabe Pharma Co., Abbivie GK, Nipponkayaku Co.Ltd, Janssen Pharmaceutical K.K. Astellas Pharma Inc., Taiho Pharmaceutical Co. Ltd., Chugai Pharmaceutical Co. Ltd., Taisho Toyama Pharmaceutical Co. Ltd., GlaxoSmithKline K.K., UCB Japan Co. Ltd., Consultant for: AbbVie, Asahi Kasei, Astellas, AstraZeneca, AYUMI, Bristol-Myers Squibb, Chugai, Daiichi Sankyo, Eisai, Eli Lilly Japan, Janssen, Mitsubishi Tanabe, Nippon Kayaku, Novartis, Pfizer Japan Inc, Taiho, Taisho Toyama, Takeda, Teijin, Consultant for: Astra Zeneca K.K., Eli Lilly Japan K.K., Novartis Pharma K.K., Mitsubishi Tanabe Pharma Co., Asahi Kasei Medical K.K., AbbVie GK, Daiichi Sankyo Co., Ltd., Bristol Myers Squibb, and Nipponkayaku Co. Ltd., Speakers bureau: Astellas Pharma Inc., Bristol Myers Squibb, Chugai Pharmaceutical Co., Ltd., Mitsubishi Tanabe Pharma Co., Pfizer Japan Inc., Santen Pharmaceutical Co., Ltd., Takeda Pharmaceutical Co., Ltd., Teijin Pharma Ltd., AbbVie GK, Asahi Kasei Pharma Corp., Taisho Toyama Pharmaceutical Co., Ltd., SymBio Pharmaceuticals Ltd., Janssen Pharmaceutical K.K., Celltrion Inc., Nipponkayaku Co. Ltd., and UCB Japan, Speakers bureau: AbbVie, Asahi Kasei, Astellas, AstraZeneca, AYUMI, Bristol-Myers Squibb, Chugai, Daiichi Sankyo, Eisai, Eli Lilly Japan, Janssen, Mitsubishi Tanabe, Nippon Kayaku, Novartis, Pfizer Japan Inc, Taiho, Taisho Toyama, Takeda, Teijin, Speakers bureau: AbbVie GK., Bristol-Myers K.K., Chugai Pharmaceutical Co. Ltd., Mitsubishi Tanabe Pharma Co., Pfizer Japan Inc., Astellas Pharma Inc, Diaichi Sankyo Co. Ltd., Eisai Co. Ltd., Sanofi K. K., Teijin Pharma Ltd., Takeda Pharmaceutical Co. Ltd., Novartis Pharma K.K., Satoshi Soen Grant/research support from: Chugai Pharmaceutical Co,. Ltd., Diaichi Sankyo Co., Ltd., Speakers bureau: Asahi Kasei Pharma Corp, Astellas Pharma Inc, Chugai Pharmaceutical Co, Ltd., Daiichi Sankyo Co., Ltd., Eli Lilly Japan K.K., Eisai Co., Ltd. Ono Pharmaceutical CO., Ltd., Takeda Pharmaceutical Co., Ltd., Pfizer., Naoki Ishiguro Grant/research support from: AbbVie, Asahi Kasei, Astellas, Chugai, Daiichi-Sankyo, Eisai, Kaken, Mitsubishi Tanabe, Otsuka, Pfizer, Takeda, and Zimmer Biomet, Consultant for: Ono, Speakers bureau: Astellas, Bristol-Myers Squibb, Daiichi-Sankyo, Eli Lilly, Pfizer, and Taisho Toyama, Hisashi Yamanaka Grant/research support from: AbbVie, Eisai, Bristol-Meyers, Novartis, Behringer, Astellas, Kaken, Nippon-Shinyaku, Pfizer, UCB, Ayumi, Ono, Daiichi-Sankyo, Taisyo-Toyama, Takeda, Tanabe-Mitsubishi, Chugai, Teijin Pharma, Torii, YLbio, Speakers bureau: Bristol-Meyers, Astellas, Pfizer, Daiichi-Sankyo, Takeda, Tanabe-Mitsubishi, Chugai, Teijin Pharma, YLbio, Sakae Tanaka Grant/ research support from: KYOCERA Corporation and Asahi Kasei Corporation, Consultant for: Amgen Astellas BioPharma K.K., KYOCERA Corporation, Pfizer and Daiichi Sankyo Co., Ltd., Speakers bureau: Asahi Kasei Corporation, Astellas Pharma Inc, Ayumi Pharmaceutical Corporation, Eisai Co., Ltd., Ono Pharmaceutical Co., Ltd., Daiichi Sankyo Co., Ltd, Taisho Toyama Pharmaceutical Co., Ltd., Mitsubishi Tanabe pharma Corporation, Chugai Pharmaceutical Co., Ltd., Teijin Pharma Ltd., Eli Lilly, Hisamitsu Pharmaceutical Co, Inc., Pfizer, Bristol-Myers., Makiko Kobayashi Employee of: Daiichi Sankyo Co., Ltd., Naoki Okubo Employee of: Daiichi Sankyo Co., Ltd., Takaya Nitta Employee of: Daiichi Sankyo Co., Ltd., Yoshiya Tanaka Grant/research support from: Abbvie, Astellas, Bristol-Myers Squibb, Chugai, Daiichi-Sankyo, Eisai, 